\section{Avaliação de conhecimentos e práticas que adultos e crianças têm acerca da doença de Chagas e seus vetores em região endêmica de Minas Gerais, Brasil}

\author{
Evaluation of knowledge and practices related to \\ Chagas disease and its vectors among adults and \\ children in an endemic region in Minas Gerais \\ State, Brazil
}

Marcos Marreiro Villela 1,2 Denise Nacif Pimenta 1 Paulo Acácio Lamounier 1 João Carlos Pinto Dias 1

\footnotetext{
${ }^{1}$ Centro de Pesquisas René Rachou, Fundação Oswaldo Cruz, Belo Horizonte, Brasil. 2 Universidade Federal de Pelotas, Pelotas, Brasil.

Correspondência J. C. P. Dias

Centro de Pesquisas René Rachou, Fundação Oswaldo Cruz.

C. P. 1743, Belo Horizonte, $M G$ 30190-012, Brasil. jcpdias@cpqrr.fiocruz.br
}

\begin{abstract}
Despite the success of the Chagas Disease Control Program (PCCD) in Brazil, some endemic areas have experienced difficulty in maintaining the program's activities, especially after the health system's decentralization, since the sustainability of control measures for Chagas disease and vectors is known to depend on information and community participation. This study aimed to analyze knowledge and practices related to vectors and Chagas disease in Bambuí, Minas Gerais State, Brasil. The population's knowledge was tested with a questionnaire, accompanied by six illustrations of triatomine bugs for identification. Both adults and primary and secondary schoolchildren in rural areas of the county participated in the research. The Bambui population showed good overall knowledge on triatomines and Chagas disease in both groups (adults and children), although the concepts were limited to preventing the insect vector from invading houses. The results emphasize the importance of educational campaigns in the context of the program as a fundamental component of community participation in Chagas disease vector control.
\end{abstract}

Chagas Disease; Disease Vectors; Communicable Disease Control; Health, Knowledge, Atitudes, Practice

\section{Introdução}

O controle de grandes endemias como a doença de Chagas ainda constitui um dos maiores desafios na Saúde Pública. O Brasil apresenta hoje uma complexa situação no que tange à profilaxia de endemias. A consolidação da educação e promoção da saúde no âmbito do Sistema Único de Saúde (SUS) requer avaliação da política de saúde direcionada para o controle de endemias transmitidas por vetores. A construção do SUS tem sido o centro desse processo, criandose possibilidades de estratégias de controle de endemias em uma síntese que compatibilize o momento epidemiológico, a descentralização e a participação da população 1 .

Com relação à doença de Chagas, do ponto de vista seja da saúde pública, seja do impacto econômico, a endemia é uma das mais importantes doenças parasitárias na América Latina, onde mais de 10 milhões de pessoas são infectadas com o parasito Trypanosoma cruzi, e aproximadamente 90 milhões de indivíduos ainda estão sob risco de contraírem a doença 2,3,4. Dados recentes demonstram que, globalmente, a doença de Chagas está associada a 14 mil mortes anuais e 0,7 milhão de anos perdidos por morte e/ou qualidade de vida (DALY - disability adjusted life years lost), constituindo-se na sexta doença tropical de maior importância no mundo 5 .

Apesar do sucesso no controle da enfermidade que alguns países obtiveram, o maior risco ao 
controle da doença de Chagas advém, ironicamente, desse sucesso. Como há necessidade de manter o controle e a vigilância epidemiológica da doença, a partir do momento em que as campanhas cumprem sua missão e a incidência da doença declina, as autoridades políticas retraem, e os financiamentos se tornam mais escassos. Habita aí o perigo de a doença tornar às áreas onde não mais existe, ou se alastrar para novas regiões 1 .

A transformação da pesquisa em ações de saúde é um processo complexo, dispendioso e, algumas vezes, extremamente demorado, como bem ilustra o sucesso da eliminação da transmissão vetorial da doença de Chagas no Cone Sul das Américas. Na região, em 1947, Dias e Pellegrino, no Brasil, e Romaña e Abalos, na Argentina, conduziram os experimentos que demonstraram a ação de inseticidas organoclorados contra os triatomíneos vetores do T. cruzi, mas só na década de 1990 foi possível conseguir a mobilização política e os recursos necessários para a Iniciativa dos Países do Cone Sul, que aplicou em larga escala, e com enorme sucesso, essa intervenção como estratégia básica de combate aos insetos vetores 6 .

A sustentabilidade das ações de prevenção e controle da doença de Chagas passa, obrigatoriamente, pela informação e participação da população. Porém, o grande problema é que no Brasil nem o Ministério da Saúde nem as Secretarias Estaduais de Saúde têm equipes bem estruturadas e atuantes no ramo educativo; também não há ações estruturadas na educação formal que priorizem as atividades pertinentes a esses agravos. O mesmo se dá no plano internacional, que, como afirma Dias 7 (p. 17): "considering the fundamental side of health education, in spite of several attempts (including in SCI [Southern Cone Initiative], for instance, in punctual efforts of Paraguay, Uruguay, Bolivia, Chile), a national and permanent educative structure has never been improved, as much as in elementary or superior school level".

Conseqüentemente, a avaliação de conhecimentos e práticas por parte das populações pode servir como instrumento facilitador para a diminuição das fronteiras da informação e promoção em saúde, sendo desafios fundamentais, de um lado, sua inclusão ou uso em políticas públicas e, de outro, sua consistência técnica, como também sua capacidade transformadora e impulsionadora. No que tange à doença de Chagas, é importante que as atividades educativas façam parte do treinamento das equipes do programa de controle, para que estas possam fornecer à população informações necessárias para a compressão da importância do trabalho de captura dos triatomíneos. No decorrer da pesquisa, devese solicitar, quando possível, a presença de um morador, a fim de orientá-lo quanto aos locais habituais de encontro dos "barbeiros" 8.

Apesar de intensas pesquisas a respeito dos aspectos biomédicos da doença, relativamente pouca informação tem sido gerada a respeito das percepções e conhecimentos sobre a doença de Chagas, infecção por T. cruzi, ou sua importância em comunidades endêmicas 9,10. Segundo Sanmartino \& Crocco 11, maior conhecimento sobre a doença de Chagas implicará importante avanço na luta contra a afecção e seus vetores, conduzindo os habitantes de áreas endêmicas à melhor compreensão de sua realidade e à aquisição de hábitos que lhes permitam ser os protagonistas do seu próprio bem estar.

O conhecimento da população sobre os triatomíneos e a doença de Chagas é de suma importância para que se promova colaboração no combate aos vetores e, assim, evite-se a transmissão vetorial da moléstia, sobretudo após a descentralização do sistema de saúde e, conseqüentemente, do Programa de Controle da Doença de Chagas (PCDCh). Além disso, as intervenções verticais, que eram realizadas anualmente, são onerosas e pouco sensíveis na detecção de triatomíneos, especialmente em residências com baixa densidade de vetores 12 . Segundo Silva et al. 13, o processo de detecção desses insetos com a participação da população, se forem conseguidas as necessárias motivação e continuidade, culmina em uma vigilância contínua, se comparada às atividades realizadas pelas equipes de campo da Fundação Nacional de Saúde (FUNASA). Uma agravante é que, como a doença de Chagas se trata de uma enfermidade cujos efeitos ou sintomatologia geralmente não são observados imediatamente, o interesse e a participação comunitária podem tornar-se escassos 14 .

Organismos internacionais de saúde, como a Organização Mundial da Saúde (OMS), na década de 1970, passaram a enfatizar a necessidade de estudar os conhecimentos prévios e o contexto cultural das populações para melhor adequação às práticas de saúde, constatando a importância da participação da comunidade local no combate às doenças endêmicas. As crenças e iniciativas da população devem ser avaliadas para se confeccionarem mensagens educativas compreensíveis e atraentes, aumentando o contingente de pessoas que auxiliam num determinado programa 15 .

Naquela mesma década, em franco período de recessão, começaram os primeiros experimentos de vigilância epidemiológica com participação comunitária no Município de Bambuí (Estado de Minas Gerais), pois, já na ocasião, os 
pesquisadores notavam a dificuldade de se manter uma atividade permanente quando a transmissão vetorial já estivesse sob controle e a densidade populacional dos insetos fosse muito baixa nos domicílios. A época era complicada, sendo o programa vertical e limitado; além disso, existia o ambiente de ditadura, sendo qualquer reunião encarada como suspeita ou subversiva 16.

Conforme Briceño-León 17, no caso das endemias, a participação comunitária tem tido importância cada vez maior, pois tais moléstias são fenômenos coletivos que afetam grande parte da população, e qualquer política de controle requer a cooperação de pessoas sob risco de contrair essas doenças. Assim, a noção de participação comunitária tem sofrido mudanças nos últimos 40 anos, as quais tendem a refletir as transformações que têm se dado dentro do Estado e suas relações com a sociedade civil 18,19 . Portanto, um elemento fundamental nos trabalhos de educação, como ponto de partida do processo pedagógico, abrange o saber anterior do educando. No trabalho, na vida social e na luta pela sobrevivência e pela transformação da realidade, as pessoas vão adquirindo entendimento sobre a sua inserção na sociedade e na natureza. Esse conhecimento fragmentado e pouco elaborado é a matéria-prima da educação popular e participação comunitária. A valorização do saber e valores do "educando" permite que ele se sinta "em casa" e mantenha sua iniciativa. Logo, não se deve reproduzir a passividade usual dos processos pedagógicos tradicionais 20 .

Novos trabalhos sobre o conhecimento da população são essenciais, como alternativa que propicie seu maior envolvimento na vigilância triatomínica, principalmente neste novo sistema descentralizado e com baixo índice populacional e de invasão dos insetos vetores. O controle e a epidemiologia da doença de Chagas apontam, cada vez mais, no rumo da participação comunitária, ao mesmo tempo em que diminuem a visibilidade da doença em áreas sob controle 1,12.

\section{Materiais e métodos}

\section{Região do estudo e população}

O conhecimento da população rural do Município de Bambuí foi avaliado com base em questionário acompanhado de seis figuras de hemípteros para identificação, três das quais eram de triatomíneos diagnosticados na região (fêmea de Panstrongylus megistus, ninfa de P. megistus e adulto de Rhodnius neglectus). Os exemplares restantes eram dois hemípteros predadores e um fitófago. Também foram realizadas questões so- bre o eventual encontro de triatomíneos, transmissão de alguma doença pelo inseto, nome da enfermidade, órgãos acometidos por esta, ações para evitar tais insetos no domicílio, sugestões para melhorar o PCDCh, dentre outros questionamentos. O Município de Bambuí foi escolhido por ter tradição no controle da endemia há mais de cinqüenta anos, somado ao fato de o Centro de Pesquisas René Rachou, da Fundação Oswaldo Cruz (CPqRR/Fiocruz), manter um posto avançado de estudos na cidade (Posto Avançado de Estudos Emmanuel Dias - PAEED). Foram avaliados os conhecimentos de crianças a partir dos dez anos (6o ao 9o ano do Ensino Fundamental), de alunos do ensino médio e da população rural adulta.

Uma vez que não existem registros de novos casos da doença na região desde 1977 21, sugerindo a interrupção da transmissão de T. cruzi há mais de trinta anos, as crianças foram escolhidas para se avaliarem quais são os conhecimentos que estes jovens estudantes têm sobre a endemia após o virtual desaparecimento da transmissão vetorial e a sensível diminuição da quantidade de triatomíneos no ambiente domiciliar. Além disso, as crianças são os melhores porta-vozes e divulgadores de um programa de controle na comunidade, visto que freqüentemente atuam como multiplicadores de conhecimento para os próprios pais $14,22,23$. As respostas ao questionário foram avaliadas estatisticamente, comparandose as das crianças e as dos adultos. Também tivemos acesso ao material educativo que era distribuído no município e em regiões adjacentes.

Foi realizado um questionário inicial em uma amostra de crianças e adultos para verificação e adequação das perguntas. Em seguida, foram procedidas breves correções para principiar o inquérito definitivo.

As unidades amostrais escolhidas para aplicação do questionário foram as escolas rurais, no caso das crianças, e os domicílios rurais, no caso dos adultos. Foram entrevistados todos os estudantes do 6o ao 9o ano do Ensino Fundamental e os do Ensino Médio das três escolas rurais de Bambuí (Escola Municipal Pau Ferro, Escola Municipal Açudinhos e Escola Municipal Lagoa Seca). Para os adultos. a unidade amostral escolhida para o inquérito foi o domicílio de várias localidades. Em cada unidade, previamente escolhida por sorteio, era entrevistado um homem ou uma mulher, tentando-se manter um número proporcional entre ambos os gêneros.

Com base no cálculo de tamanho da amostra determinado pela OMS 24, avaliou-se ser necessária uma amostra de 310 pessoas (entre crianças e adultos) para a aplicação do questionário, admitindo-se um erro de $5 \%$, a fim de se abranger nú- 
mero significativo de entrevistados para ambos os grupos. Caso o domicílio estivesse fechado ou os responsáveis não se encontrassem, a entrevista seria realizada no domicílio mais próximo. Um dos problemas encontrados foi que inúmeros croquis (mapas) referentes à zona rural do município estavam desatualizados, com boa parte das casas descritas fechadas ou inexistentes; além disso, muitas construções novas não estavam relacionadas, por isso o inquérito foi realizado no maior número de domicílios possível em cada localidade. Por vezes, a atualização dos croquis era realizada pelo agente municipal responsável pelo combate aos vetores da doença de Chagas, quando o mesmo acompanhava o trabalho de campo e guiava-nos na zona rural. A participação na pesquisa foi voluntária, sendo antecedida pela assinatura de termo de consentimento informado e autorizada pelo Comitê de Ética do CPqRR/Fiocruz. As informações coletadas foram digitadas e analisadas em planilha do programa Excel (Microsoft Corp., Estados Unidos); foi utilizado também o programa estatístico Minitab versão XIII (Minitab Inc., State College, Estados Unidos). Para se comparar a existência de associação entre as variáveis categóricas, utilizou-se o teste qui-quadrado.

Durante o período das entrevistas (junho de 2006 até março de 2007), após a aplicação de questionário, foram feitos esclarecimentos aos moradores sobre os vetores e a doença de Chagas. Enfocaram-se medidas de controle dos insetos, como a adequada organização do intra e peridomicílio e o destino correto a ser dado aos "barbeiros" que invadissem esses ambientes. Em algumas oportunidades, também se realizaram explanações coletivas (palestras em colégios).

\section{Resultados}

Quanto ao conhecimento da população sobre os triatomíneos e a doença de Chagas, foram entre-

\section{Tabela 1}

Capacidade de reconhecimento pela população de triatomíneos no mostruário. Bambuí, Minas Gerais, Brasil.

\begin{tabular}{lcccc}
\hline $\begin{array}{l}\text { Sabe reconhecer } \\
\text { os barbeiros? }\end{array}$ & $\mathbf{n}$ & Adultos & \multicolumn{2}{c}{ Crianças } \\
\hline Sim & 155 & 89,1 & $\mathbf{n}$ & $\%$ \\
Não & 19 & 10,9 & 46 & 66,7 \\
Total & 174 & 100,0 & 138 & 33,3 \\
& & & 100,0 \\
\hline
\end{tabular}

vistados 174 adultos (95 homens; 79 mulheres) e 138 escolares (73 homens; 65 mulheres). Notou-se que tanto os adultos quanto as crianças apresentam bom conhecimento em relação aos triatomíneos, entretanto os primeiros reconhecem melhor os insetos ( $\mathrm{p}<0,05)$, não havendo diferença entre os gêneros (masculino e feminino) para ambos os grupos ( $p>0,05$ ) (Tabela 1). Cabe apontar que não houve diferença significativa quando as crianças foram avaliadas em dois grupos: estudantes do Ensino Fundamental e estudantes do Ensino Médio ( $p>0,05$ ), embora a maioria dos escolares cursasse o Ensino Fundamental (99 alunos). Diante do mostruário, quando o entrevistado apontou um triatomíneo e outro hemíptero não hematófago, a pessoa questionada foi classificada como sabendo reconhecer os triatomíneos, entretanto isso só ocorreu em seis oportunidades (dois adultos e quatro crianças).

O inseto mais apontado no mostruário foi P. megistus fêmea, tanto pelos adultos $(68,4 \%)$, como pelos estudantes (63,8\%). Quanto ao reconhecimento de ninfas, este foi estatisticamente igual entre adultos $(9,8 \%)$ e crianças $(6,5 \%)$.

Quando questionados sobre se encontraram triatomíneos em sua casa nos últimos dois anos, $21,3 \%$ (37) dos adultos e 14,5\% (20) das crianças responderam afirmativamente. Quanto à atitude adotada após o encontro do inseto, a maioria dos adultos levou o inseto suspeito até um Posto de Informação de Triatomíneos (PIT) (normalmente sediado em escolas ou postos de saúde) ou entregou para o agente do PCDCh municipal (25 casos), entretanto dois deles não tomaram nenhuma atitude, dois colocaram o inseto em um recipiente, mas não deram o destino correto a este, e outros oito mataram o inseto. No que diz respeito às crianças, nove levaram o inseto suspeito até um PIT ou entregaram para o agente, oito mataram o triatomíneo e três disseram não ter tomado nenhuma providência.

Uma pergunta que representa a importância histórica da doença de Chagas em Bambuí foi se o entrevistado ou alguém da família já havia sido picado pelo "barbeiro". Os adultos responderam afirmativamente em 48,3\% (84) dos casos, sendo os pais os parentes mais citados $(36,9 \%)$, seguido dos irmãos (16,7\%), cônjuge $(10,7 \%)$, avós $(5,9 \%)$ e outros $(9,6 \%)$. É primordial relatar que $17(20,2 \%)$ entrevistados relataram que eles próprios já haviam sido picados. Focando nas crianças, essa proporção foi menor, já que $24,6 \%$ (34 casos) disseram apresentar familiares que já haviam sido picados pelos triatomíneos, sendo os avós os parentes mais citados $(61,8 \%)$, seguido dos tios $(11,8 \%)$ e pais $(8,8 \%)$. Nenhuma criança comentou ter sido picada pelo triatomíneo. 
Na pergunta sobre se aquele inseto poderia transmitir alguma doença, 165 (94,8\%) adultos e $125(90,6 \%)$ crianças responderam afirmativamente. Logo após, foi questionado qual o nome da doença transmitida (Tabela 2). Para os que responderam que os insetos não transmitem doença, foi explicado que, na realidade, estes podem transmitir uma enfermidade, e passou-se a ler as alternativas, sendo assinaladas estas respostas como induzidas, dando-se seguimento à entrevista. Para aqueles que disseram esquecer o nome da doença mesmo sabendo que o inseto transmitia, também foram lidas alternativas, sendo as respostas também consideradas induzidas.

Não houve diferença significativa entre a proporção de adultos e estudantes que falaram ser a doença de Chagas a enfermidade que podia ser transmitida pelos insetos $(p>0,05)$. Entre os adultos o índice de pessoas induzidas que disseram doença de Chagas (aqueles para quem foi necessário ler as alternativas) atingiu $30,6 \%$, ao passo que, entre as crianças, o índice ficou em $21,8 \%$, sendo significativamente menor do que entre os adultos $(\mathrm{p}<0,05)$. Quando comparamos apenas os entrevistados não induzidos, não houve diferença estatisticamente significativa ( $p>0,05)$, demonstrando, neste caso, que o fator indução não influenciou estatisticamente nas respostas.

Foi interpelado se a doença pode se tornar grave, a que $95,4 \%$ (166) da população adulta e 90,6\% (125) dos escolares responderam afirmativamente.

Com relação aos órgãos que a doença pode acometer, o coração foi o mais citado em ambos os grupos avaliados, todavia foi estatisticamente mais lembrado pelos adultos $(84,5 \%)$ quando comparado aos estudantes $(68,8 \%)(\mathrm{p}<0,05)$. $\mathrm{O}$ intestino e o esôfago foram esquecidos pela maioria da população. Órgãos com pouca ou nenhuma importância na doença de Chagas, como o fígado, estômago, rins, e olhos, também figuraram nas respostas. As alternativas foram lidas para $45,9 \%$ dos adultos e $27,5 \%$ dos estudantes, havendo diferença significativa no que tange à indução $(\mathrm{p}<0,05)$.

Outra questão investigava sobre o que fazer para se evitarem "barbeiros" em casa. Percebeuse que tanto os adultos quanto as crianças associam a ação contra os triatomíneos, primeiramente, à idéia de limpeza e higiene. Os adultos citaram a borrifação do domicílio como segunda alternativa, enquanto as crianças comentaram que se deve evitar bagunça e amontoados em casa (Tabela 3). Importante alegar que todas as respostas foram citadas espontaneamente, não havendo alternativas, e, conseqüentemente, indução para esta questão.
Tabela 2

Respostas da população quando questionada sobre o nome da doença que os insetos podem transmitir. Bambuí, Minas Gerais, Brasil.

\begin{tabular}{lcccc}
\hline Doença & \multicolumn{2}{c}{ Adultos } & \multicolumn{2}{c}{ Crianças } \\
& $\mathbf{n}$ & $\%$ & $\mathbf{n}$ & $\%$ \\
\hline Doença de Chagas & 160 & 91,9 & 133 & 96,4 \\
Dengue & 1 & 0,6 & 3 & 2,2 \\
Febre amarela & - & - & 2 & 1,4 \\
Malária & 1 & 0,6 & - & - \\
Xistose & 1 & 0,6 & - & - \\
Não sabe, mesmo lendo & 11 & 6,3 & - & 100,0 \\
as alternativas & & & 138 & \\
Total & 174 & 100,0 & & \\
\hline
\end{tabular}

Exclusivamente aos adultos, foram realizadas duas perguntas. A primeira questionava se a pessoa sabia da existência do PCDCh no município e a outra era relativa a alguma sugestão que ela teria para melhorar o programa em razão do convívio que os adultos tiveram com este no passado. Dos entrevistados, 30,5\% não sabiam da existência do PCDCh no município, e 73,5\% das pessoas não mencionaram sugestões para melhoria do programa. Entretanto, 46 pessoas fizeram comentários, e as principais sugestões relacionaram-se com as seguintes necessidades: de o agente passar nas residências com mais freqüência (31), de pulverizar as casas (7) e de fazer palestras para a população (3).

\section{Discussão e conclusões}

Os sistemas de vigilância epidemiológica não podem prescindir da participação efetiva da população, não somente por razões de economia e eficácia, mas principalmente pelo potencial que se cria para as atividades correspondentes, além da maior interação social 25 . A participação comunitária não é algo que se imponha por meio de ações verticais ou pré-estabelecidas e deve se basear em problemas, peculiaridades e conhecimentos da própria comunidade-alvo 18. Segundo Rojas-de-Arias 26, pouco se sabe a respeito do conhecimento que a população tem da doença de Chagas, por isso avaliações sobre esse conhecimento, sobre atitudes e perspectivas dos habitantes em face da endemia, são essenciais.

Para a verificação realizada entre a população de Bambuí, de modo geral observou-se que tanto os adultos quanto as crianças apresentam bom conhecimento em relação aos triatomíne- 
Respostas da população sobre o que fazer para evitar barbeiros em casa. Bambuí, Minas Gerais, Brasil.

\begin{tabular}{|c|c|c|c|c|}
\hline \multirow[t]{2}{*}{ que fazer } & \multicolumn{2}{|c|}{ Adultos } & \multicolumn{2}{|c|}{ Crianças } \\
\hline & $\mathbf{n}$ & $\%$ & $\mathbf{n}$ & $\%$ \\
\hline Higiene, limpeza, evitar sujeira, lixo & 92 & 52,9 & 56 & 40,6 \\
\hline Borrifar o domicílio, jogar veneno, remédio & 44 & 25,3 & 8 & 5,8 \\
\hline Tapar rachaduras, gretas, rebocar frestas & 9 & 5,2 & 5 & 3,6 \\
\hline Evitar bagunça, amontoados & 12 & 6,9 & 18 & 13,0 \\
\hline Procurar, limpar galinheiro & 14 & 8,0 & 4 & 2,9 \\
\hline Olhar colchão, cama & 3 & 1,7 & 4 & 2,9 \\
\hline Procurar cômodos & 3 & 1,7 & 5 & 3,6 \\
\hline Agente passar mais & - & - & 2 & 1,4 \\
\hline Outros & 5 * & 3,4 & 8 ** & 5,8 \\
\hline Não sabe & 32 & 18,4 & 50 & 36,2 \\
\hline
\end{tabular}

* Evitar animais, desligar a luz, passar cal na casa, vedar as janelas, tirar monte de lenhas;

** Olhar casa abandonada, chiqueiro, lenhas, deixar entrar sol, arejar a casa, limpar horta, não comer tatu, olhar no paiol.

os, com porcentagens de acerto bastante satisfatórias quanto ao reconhecimento dos insetos (89,1\% para os adultos e $66,7 \%$ para os escolares). Os resultados foram superiores aos encontrados para uma análise realizada com um grupo de adultos assentados no Estado de São Paulo, em que $68,7 \%$ dos entrevistados não reconheceram o vetor 27,28. Em nossa observação, os adultos reconheceram melhor os insetos quando comparados às crianças, fato que possivelmente está relacionado com a relevância que a moléstia teve no passado, quando eram encontrados triatomíneos domiciliados na maioria das casas do ambiente rural, por vezes capturando-se milhares de exemplares 29,30. Da mesma forma, pode-se entender a ausência de diferença significativa entre o conhecimento de homens e mulheres, uma vez que ambos os grupos estiveram igualmente expostos aos insetos em décadas passadas, sobretudo durante a infância. Atualmente, o número de hemípteros hematófagos capturados no município é reduzido, e a transmissão vetorial da doença perdeu a importância de outrora; logo, evidencia-se menor conhecimento da morfologia dos "barbeiros" pelas crianças. Como os adultos entrevistados apresentavam índice de estudo geral inferior ao das crianças, mas denotaram ter melhor conhecimento sobre os triatomíneos, não houve correlação entre o índice de estudo e o reconhecimento dos insetos ( $p>0,05)$.

O inseto mais apontado como "barbeiro" foi P. megistus adulto, tanto pelos adultos, como pelos escolares, evento já esperado para o município, visto que tal espécie sempre apresentou importância epidemiológica na região, mesmo antes da ocorrência de Triatoma infestans 29,31,32. Os dois grupos apontaram a ninfa de $P$. megistus em menos de $10 \%$ das vezes, possivelmente em virtude da baixa divulgação como possível vetor que esta forma evolutiva apresenta. Ao avaliarse o material educativo distribuído em Bambuí e em municípios vizinhos, houve ausência de ilustrações de ninfas em 100\% dele. É essencial a confecção de um material educativo/informativo correto e elucidativo para a população, uma vez que, segundo García-Zapata 22 , este é o meio de difusão mais efetivo para a população, sobretudo quando associado a um programa educativo nas escolas.

A produção de materiais educativos/informativos de qualidade, como manuais, cartilhas, folhetos, cartazes e vídeos, podem servir como instrumentos auxiliares, contribuindo nas políticas públicas que visam à promoção da saúde junto à população como um todo. Para que tais materiais possam servir como recursos pedagógicos efetivos, é necessário que eles sejam elaborados dentro de critérios interdisciplinares, compatíveis com a complexidade do seu público e da temática ${ }^{33}$. De acordo com Paulo Freire 34,35,36, isso requer uma ação pedagógica transdisciplinar que inclua aspectos cognitivos, sociais, culturais e afetivos. Esses materiais devem se inserir em projetos pedagógicos inclusivos, que se fundamentem nas relações entre as pessoas, em suas atitudes, permeadas pela parceria e solidariedade 37,38 .

Quanto às práticas adotadas após o encontro dos hemípteros, os adultos tomaram a atitude correta proporcionalmente mais vezes $(67,6 \%)$ 
que as crianças (45\%), o que, possivelmente, decorre da maior experiência que os adultos apresentam quanto às normas de notificação preconizadas pelo PCDCh. Nota-se que as crianças devem ser devidamente incluídas na participação comunitária para o controle da doença de Chagas e de outras endemias, uma vez que elas se conscientizam mais facilmente que os adultos quanto às mudanças de hábitos e ficam entusiasmadas para auxiliar no programa 22,39.

Segundo trabalho clássico de Dias \& Garcia 25, o meio de comunicação entre as pessoas e o órgão executivo central pode ser múltiplo e variado, dependendo das condições e possibilidades de cada um. Assim sendo, para os dias de hoje, faculta-se a possibilidade da participação de membros do Programa Saúde da Família (PSF) no recebimento das notificações quando da visita aos domicílios, podendo este outro programa servir como aliado no controle de vetores da doença de Chagas.

Uma pergunta que balizou a importância histórica da moléstia no município era se o entrevistado ou alguém da família já havia sido picado pelo "barbeiro"; 48,3\% dos adultos e $24,6 \%$ das crianças responderam afirmativamente, sendo significativamente maior para os adultos certamente por causa da maior ocorrência da doença no passado. Vale ressaltar que os pais $(36,9 \%$ das vezes) foram os parentes mais citados pelos adultos, e 20,2\% destes relataram já terem sido picados, mostrando a maior proximidade que as pessoas tinham com os vetores e, por conseqüência, com T. cruzi. Quanto às crianças, torna-se clara a ausência de transmissão da doença nas últimas décadas no município, já que os avós foram os parentes mais citados $(61,8 \%)$.

Considerando-se o nome da doença, mais de 90\% dos adultos e das crianças responderam ser a doença de Chagas a moléstia transmitida pelo triatomíneo, demonstrando, nos dois grupos, alta associação entre o inseto e a enfermidade. Os dados encontrados são superiores aos obtidos por Verdú \& Ruiz ${ }^{40}$, que, ao questionarem sobre o nome da enfermidade a uma comunidade Guaraní na Bolívia, diagnosticaram índice de acerto de apenas 14,3\%. Contudo, na nossa avaliação, para $30,5 \%$ dos adultos, foi necessária a leitura das alternativas (questionário induzido), taxa significativamente maior do que a verificada entre as crianças, denotando maior grau de esquecimento dos adultos, alguns com idade superior a 70 anos e moradores contínuos da zona rural, onde as informações costumam ser escassas.

Merece consideração o fato de mais de $90 \%$ de todos os entrevistados considerarem que a enfermidade em questão pode se tornar grave, além de muitos acrescentarem que a doença tem capacidade de matar. Tal índice é próximo ao de um estudo procedido no Estado de Goiás, no qual $86,4 \%$ dos entrevistados comentaram que a doença de Chagas é um grave problema de saúde ${ }^{9}$. Aqui se constata que a tripanossomíase americana geralmente é vista como doença incapacitante e, por vezes, como enfermidade que resulta em morte. Nossos achados estão de acordo com o trabalho realizado por Uchoa et al. ${ }^{41}$ no mesmo município, investigação em que os entrevistados relataram que ser soropositivo para a doença de Chagas significa se sentir vulnerável por causa das limitações e da ameaça de morte súbita e imprevisível resultantes da doença.

Uma questão importante relacionou-se sobre o que fazer para evitar os triatomíneos em casa. Ressalte-se que, neste caso, não foi lida nenhuma alternativa, logo inexistiram respostas induzidas. Tanto os adultos quanto as crianças se referiram primeiramente à idéia de limpeza e higiene para evitar os hemípteros em casa, sugestão esta genérica e pouco específica para os vetores da doença de Chagas. Para os adultos, como segunda opção, citou-se a borrifação domiciliar, mencionada por vezes como "jogar veneno" ou "jogar remédio". A lembrança de tal atitude provavelmente está associada à longa história de desinsetizações do município 29,32. Já as crianças, que não acompanharam a fase de ataque (borrifações) aos domicílios infestados por "barbeiros", a segunda alternativa eleita foi "evitar bagunça ou amontoados dentro de casa”, sugestão também apropriada no combate aos hemípteros, principalmente por reduzir o número de esconderijos onde os vetores podem constituir colônias. As crianças falaram na borrifação dos domicílios como terceira alternativa, embora em índices inferiores aos encontrados para escolares de uma zona endêmica do Peru 33. Outras medidas essenciais ao encontro ou eliminação dos insetos foram mencionadas em ambos os grupos (procurar/limpar galinheiros, vistoriar cômodos, colchão e cama, rebocar frestas), entretanto essas sugestões, em ambos os grupos, não somaram mais de $8 \%$ das respostas, o que denota a necessidade de atividades educativas, já que muitas recomendações fundamentais para combater os triatomíneos foram esquecidas. Preocupante o fato de 18,4\% dos adultos e $36,2 \%$ das crianças não saberem apontar alguma atitude para se evitar/combater os vetores, mesmo num município exemplar no que se refere ao controle da doença de Chagas, evidenciando escassez de informações. Portanto, no que diz respeito às atitudes e práticas para combater os triatomíneos, o conhecimento da população mostrou-se limitado. 
Nas duas perguntas feitas exclusivamente aos adultos, um dado alarmante foi o desconhecimento de boa parcela da população de Bambuí sobre a existência do PCDCh no município; em adição, em algumas oportunidades, as pessoas relataram fazer muitos anos que o agente não realizava a pesquisa em sua morada. Esse dado foi corroborado quando analisadas as sugestões que a população referiu para melhorar o Programa: das 46 pessoas que se manifestaram, $67,3 \%$ mencionaram a necessidade de o agente passar mais freqüentemente como primeira alternativa, seguida do ato de borrifar as casas (15,2\%), o que expõe a carência deste serviço em algumas localidades. Fazer palestras para a população foi a terceira sugestão mais apontada, que, mesmo ocorrendo em apenas três oportunidades, indica a necessidade de mais trabalho educativo para a comunidade. Com os dados observados, notou-se como o agente que trabalha no programa passa a ser referência sobre o assunto. Logo, é fundamental que ele realize palestras sobre a doença de Chagas e esclareça como a população pode participar/auxiliar na vigilância entomológica da enfermidade 18 .

Ao final das entrevistas foram dadas explicações para todos os entrevistados sobre a prevenção da doença de Chagas e a forma correta de participar do controle dos "barbeiros". Em adição, os pesquisadores se colocaram à disposição para dirimir quaisquer dúvidas que surgissem em relação à doença. Estuda-se a possibilidade de confeccionar um material abrangendo questões básicas da moléstia, com maior divulgação dos PITs e informações sobre como a população pode contribuir no combate aos insetos vetores.
Segundo Winch et al. 42, as abordagens que utilizam a participação da comunidade no delineamento e encaminhamento de soluções para os problemas de saúde pública oferecem vantagens em relação aos programas tradicionais de controle de vetores.

\section{Considerações finais}

Embora os resultados demonstrem, de modo geral, que o conhecimento sobre a endemia chagásica em Bambuí esteja relativamente disseminado, os dados ressaltam a necessidade de atividades educativas no contexto do programa, como elemento basal para a melhoria da detecção e eliminação dos triatomíneos do ambiente domiciliar. Essa ação, além de permitir uma vigilância sustentável, pode promover mudanças permanentes nos hábitos e atitudes das pessoas em face da endemia. Para avaliações futuras, julgamos que pesquisas qualitativas sobre o tema contribuiriam para o planejamento de ações educativas na região e permitiriam um aprofundamento dos dados colhidos.

Ressalta-se que, mesmo o Município de Bambuí sendo um dos que apresentou o PCDCh mais regular e ativo, se considerarmos a totalidade da região, contando com grande importância histórica no controle da doença, boa parte dos entrevistados desconhecia o PCDCh. Portanto, trabalhos da mesma natureza podem ser elaborados em outros municípios, já que é possível ser escassa e preocupante a situação que no tange ao conhecimento e práticas adotadas na prevenção da doença de Chagas em diversas outras localidades rurais. 


\section{Resumo}

Apesar do sucesso no Programa de Controle da Doença de Chagas (PCDCh) no Brasil, alguns locais encontram dificuldades na manutenção do programa, sobretudo após a descentralização do setor saúde. Sabe-se que a sustentabilidade das ações de controle dos vetores da doença de Chagas depende da informação e participação comunitária. O presente trabalho avaliou os conhecimentos e práticas que a população de Bambuí, Minas Gerais, apresenta sobre os triatomíneos e a doença de Chagas. O conhecimento foi testado por meio da aplicação de questionário acompanhado de seis figuras de hemípteros para identificação. Participaram da pesquisa tanto adultos, como crianças dos ensinos fundamental e médio das escolas rurais presentes no município. Os dados apontaram que a população de Bambuí tem bons conhecimentos gerais sobre os triatomíneos e a doença de Chagas em ambos os grupos (adultos e crianças), entretanto os conceitos se mostraram limitados quanto ao que fazer para evitar esses vetores invadindo domicílios. Os resultados ressaltam a necessidade de campanhas educativas no contexto do PCDCh como elemento fundamental da participação comunitária no combate aos triatomíneos.

Doença de Chagas; Vetores de Doenças; Controle de Doenças Transmissiveis; Conhecimentos, Atitudes e Práticas em Saúde

\section{Referências}

1. Dias JCP. Participação, descentralização e controle de endemias no Brasil. In: Barata RB, BriceñoLeón R, organizadores. Doenças endêmicas: abordagens sociais, culturais e comportamentais. Rio de Janeiro: Editora Fiocruz; 2000. p. 269-97.

2. Miles MA. The discovery of Chagas disease: progress and prejudice. Infect Dis Clin North Am 2004; 8:247-60.

3. World Health Organization. Chagas disease information. The UNICEF-UNDP-Programme on TDR http://www.who.int/tdr/diseases/chagas/ (acessado em 30/Jan/2006).

4. Maguire JH. Chagas disease: can we stop the deaths? N Engl J Med 2006; 355:760-1.

5. Hotez P. Incorporating a rapid-impact package for neglected tropical diseases with programs for HIV/ AIDS, tuberculosis, and malaria. PLoS Med 2006; 3 : e102.

6. Morel CM. Chagas disease, from discovery to control - and beyond: history, myths and lessons to take home. Mem Inst Oswaldo Cruz 1999; 94 Suppl I:3-16.

7. Dias JCP. Southern Cone Initiative for the elimination of domestic populations of Triatoma infestans and the interruption of transfusional Chagas disease. Historical aspects, present situation, and perspectives. Mem Inst Oswaldo Cruz 2007; 102:11-8.

\section{Colaboradores}

M. M. Villela executou a pesquisa no campo, interpretou os dados e redigiu o artigo. D. N. Pimenta auxiliou na compilação do projeto e realizou a revisão crítica do trabalho. P. A. Lamounier colaborou na elaboração do projeto e na coleta dos dados no campo e realizou a revisão crítica do trabalho. J. C. P. Dias orientou a pesquisa, além de contribuir na redação e revisão final do artigo.

\section{Agradecimentos}

Estudo realizado com apoio financeiro do Programa Especial de Pesquisas e Treinamento em Doenças Tropicais - TDR (Organização Mundial da Saúde/Banco Mundial/Programa das Nações Unidas para o Desenvolvimento).
8. Wanderley DMV. Perspectivas de controle da doença de Chagas no Estado de São Paulo [Tese de Doutorado]. São Paulo: Faculdade de Saúde Pública, Universidade de São Paulo; 1994.

9. Williams-Blangero S, VandeBerg JL, Teixeira ARL Attitudes towards Chagas' disease in an endemic Brazilian community. Cad Saúde Pública 1999; 15:7-13.

10. Hueb MFD, Loureiro SR. Revisão: aspectos cognitivos psicossociais associados a doença de Chagas. Psicol Estud 2005; 10:137-42.

11. Sanmartino M, Crocco L. Conocimientos sobre la enfermedad de Chagas y factores de riesgo en comunidades epidemiológicamente diferentes de Argentina. Rev Panam Salud Pública 2000; 7:173-7.

12. Dias JCP. Chagas disease control in Brazil: which strategy after the attack phase? Ann Soc Belg Med Trop 1991; 71:75-86.

13. Silva RA, Bonifácio PR, Wanderley DMV. Doença de Chagas no Estado de São Paulo: comparação entre pesquisa ativa de triatomíneos em domicílios e notificação de sua presença pela população em área de vigilância entomológica. Rev Soc Bras Med Trop 1999; 32:653-9. 
14. Crocco L, Rodríguez C, Catalá S, Nattero J. Enfermedad de Chagas en Argentina: herramientas para que los escolares vigilen y determinen la presencia de factores de riesgo en sus viviendas. Cad Saúde Pública 2005; 21:646-51.

15. Vasconcelos EM. Educação popular como instrumento de reorientação das estratégias de controle das doenças infecciosas e parasitárias. Cad Saúde Pública 1998; 14 Suppl 2:39-57.

16. Dias JCP. Problemas e possibilidades de participação comunitária no controle das grandes endemias do Brasil. Cad Saúde Pública 1998; 14 Suppl 2:19-37.

17. Briceño-León R. A cultura da enfermidade como fator de proteção e de risco. In: Veras RP, Barreto ML, Almeida Filho N, Barata RB, organizadores. Epidemiologia: contextos e pluralidade. Rio de Janeiro: Editora Fiocruz/ABRASCO; 1998. p. 121-31.

18. Dias JCP. Participação comunitária nos programas de saúde. Rev Bras Malariol Doenças Trop 1986; 38:103-10.

19. Briceño-León R. El contexto político de la participación comunitaria en América Latina. Cad Saúde Pública 1998; 14 Suppl 2:141-7.

20. Vasconcelos EM. Educação popular: de uma prática alternativa a uma estratégia de gestão participativa das políticas de saúde. Physis (Rio J.) 2004; 14:67-83.

21. Lima-Costa MFF, Barreto SM, Guerra HL, Firmo JOA, Uchoa E, Vidigal PG. Ageing with Trypanosoma cruzi infection in a community where the transmission has been interrupted: the Bambuí Health and Ageing Study (BHAS). Int J Epidemiol 2001; 30:887-93.

22. García-Zapata MTA. A divulgação científica no controle de doenças tropicais: um ponto de vista. Comunicação \& Sociedade 1991; 10:103-14.

23. Schall V, Diniz MCP. Information and education in schistosomiasis control: an analysis of the situation in the state of Minas Gerais, Brazil. Mem Inst Oswaldo Cruz 2001; 96:35-43.

24. World Health Organization. Adequacy of sample size in health studies. Geneva: World Health Organization; 1990.

25. Dias JCP, Garcia ALR. Vigilância epidemiológica com participação comunitária. Revista Institucional en Educación para la Salud 1976; 19:29-44

26. Rojas-de-Arias A. Social and epidemiological determinants of Chagas disease: basic information for a surveillance and control policy in the Southern Cone. Mem Inst Oswaldo Cruz 2007; 102 Suppl I:19-21.

27. Silva RA, Sampaio SMP, Poloni M, Koyanagui PH, Carvalho ME, Rodrigues VLCC. Pesquisa sistemática positiva e relação com conhecimento da população de assentamento e reassentamento de ocupação recente em área de Triatoma sordida (Hemiptera, Reduviidae) no Estado de São Paulo, Brasil. Cad Saúde Pública 2004; 20:555-61.

28. Silva RA, Sampaio SMP, Poloni M, Reis G, Koyanagui PH, Carvalho ME, et al. Conhecimento sobre doença de Chagas de população moradora em assentamentos rurais de ocupação recente e antiga, Estado de São Paulo, Brasil. Rev Soc Bras Med Trop 2004; 37 Suppl III:23.
29. Dias E. Um ensaio profilático de moléstia de Chagas. Rio de Janeiro: Imprensa Nacional; 1945.

30. Dias E, Pinto OS. Combate aos triatomas com BHC na cidade de Bambuí, Minas Gerais, Brasil. Rev Bras Malariol Doenças Trop 1952; 4:22-46.

31. Dias E, Pellegrino J. Alguns ensaios com o gammexane no combate aos transmissores da doença de Chagas. Bras Med 1948; 62:185-91.

32. Dias JCP. Doença de Chagas em Bambuí, Minas Gerais, Brasil. Estudo clínico-epidemiológico a partir da fase aguda entre 1940 e 1982 [Tese de Doutorado]. Belo Horizonte: Universidade Federal de Minas Gerais; 1982.

33. Luz ZMP, Pimenta DN, Rabello A, Schall V. Evaluation of informative materials on leishmaniasis distributed in Brazil: criteria and basis for the production and improvement of health education materials. Cad Saúde Pública 2003; 19:561-9.

34. Freire P. Pedagogia do oprimido. Rio de Janeiro: Editora Paz \& Terra; 1987.

35. Freire P. Pedagogia da esperança: um reencontro com a pedagogia do oprimido. Rio de Janeiro: Editora Paz \& Terra; 1994.

36. Freire P. Pedagogia da autonomia: saberes necessários à prática educativa. Rio de Janeiro: Editora Paz \& Terra; 1999.

37. Schall VT, Modena CM. As novas tecnologias de informação e comunicação em educação em saúde. In: Minayo MCS, Coimbra Jr. CEA, organizadores. Críticas e atuantes: ciências sociais em saúde na América Latina. Rio de Janeiro: Editora Fiocruz; 2005. p. 245-55.

38. Struchiner M, Giannella TR, Ricciardi RV. Novas tecnologias de informação e educação em saúde diante da revolução comunicacional e informacional: In: Minayo MCS, Coimbra Jr. CEA, organizadores. Críticas e atuantes: ciências sociais em saúde na América Latina. Rio de Janeiro: Editora Fiocruz; 2005. p. 257-72.

39. Cabrera R, Mayo C, Suárez N, Infante C, Náquira C, García-Zapata MTA. Conocimientos, actitudes y prácticas sobre la enfermedad de Chagas en población escolar de una zona endémica del Perú. Cad Saúde Pública 2003; 19:147-54.

40. Verdú J, Ruiz MT. Control del Chagas en comunidades guaraníes: conocimiento y hábitos higiénicos dentro del Proyecto de Mejoramiento de Viviendas en Bolívia. Gac Sanit 2003; 17:166-8.

41. Uchôa E, Firmo JOA, Dias EC, Pereira MSN, Gontijo ED. Signos, significados e ações associados à doença de Chagas. Cad Saúde Pública 2002; 18: 71-9.

42. Winch P, Kendall C, Gubler D. Effectiveness of community participation in vector borne-disease control. Health Policy Plan 1992; 7:1-9.
Recebido em 06/Mai/2008
Versão final reapresentada em 23/Mar/2009
Aprovado em 13/Abr/2009 